\title{
Jacobi Polynomial Expansions of a Generalized Hypergeometric Function over a Semi-Infinite Ray
}

\author{
By Y. L. Luke and J. Wimp
}

1. Introduction. Suppose $f(x)$ is continuous and has a piecewise continuous derivative for $0 \leqq x / \lambda \leqq 1$. Then $f(x)$ may be expanded into a uniformly convergent series of shifted Jacobi polynomials in the form

$$
\begin{aligned}
f(x)=\sum_{n=0}^{\infty} a_{n}(\lambda) R_{n}{ }^{(\alpha, \beta)}(x / \lambda), & \\
& \leqq x / \lambda \leqq 1-\epsilon, \epsilon>0 ; \alpha>-1, \quad \beta>-1,
\end{aligned}
$$

where $R_{n}{ }^{(\alpha, \beta)}(x)=P_{n}{ }^{(\alpha, \beta)}(2 x-1)$ and the latter is the usual notation for the Jacobi polynomial [1, Ch. 10]. Various techniques are available for the determination of the coefficients $a_{n}(\lambda)$. In this connection, see, for example, the references $[2,3,4,5,6,7]$.

Suppose that $f(x)$ satisfies the above conditions for $1 \leqq x / \lambda \leqq \infty$ where $|\arg \lambda|<\varphi$. Then we may write

$$
\begin{aligned}
f(x)=\sum_{n=0}^{\infty} b_{n}(\lambda) R_{n}{ }^{(\alpha, \beta)}(\lambda / x) & \\
& \leqq \lambda / x \leqq 1-\epsilon, \quad \epsilon>0 ; \quad \alpha>-1, \quad \beta>-1 .
\end{aligned}
$$

If $f(x)$ has an asymptotic expansion of the form

$$
f(x) \sim \sum_{n=0}^{\infty} c_{n} x^{-n}, \quad x \rightarrow \infty, \quad|\arg x|<\varphi,
$$

then (1.2) may be interpreted as a summability process which converts the generally divergent expansion (1.3) into a convergent expansion. If $f(x)$ in (1.3) is of hypergeometric type, ${ }^{*}$ then the coefficients $b_{n}(\lambda)$ may be found formally at least using the procedures [5,6]. These yield for $b_{n}(\lambda)$ an asymptotic series in $\lambda$ which is also of hypergeometric type. The asymptotic representation for $b_{n}(\lambda)$ in general is not suitable for computation. We are confronted with two problems: one is the interpretation of the asymptotic series for $b_{n}(\lambda)$, and the other is the computation of $b_{n}(\lambda)$.

In this paper, we show how both problems can be solved for a confluent hypergeometric function. Actually we derive a representation for $b_{n}(\lambda)$ when $f(x)$ is the $G$-function, which includes the confluent hypergeometric function as a special case. Our computational scheme for $b_{n}(\lambda)$ is exhibited only when $f(x)$ is a confluent hypergeometric function, although the ideas involved can be extended to cover other special cases of the $G$-function as well.

In Section II, we prove an expansion theorem of the form (1.2) when $f(x)$ is the

Received March 29, 1963.

* For the definition and properties of generalized hypergeometric series including the $G$ function as well as other notations used in this paper, see [1, Chs. 4, 5, 6]. 
$G$-function and show how both convergent and asymptotic representations for $b_{n}(\lambda)$ may be derived. These results are specialized in Section III for the case when $f(x)$ is a confluent hypergeometric function, and in Section IV it is shown how $b_{n}(\lambda)$ may be computed by a recursion scheme. Finally, in Section V, we tabulate coefficients for the cases where $R_{n}{ }^{(\alpha, \beta)}(x)$ is the shifted Chebyshev polynomial and $f(x)$ is the error function, the exponential, sine and cosine integrals, and the Bessel functions $K_{0}(x)$ and $K_{1}(x)$.

2. Expansion of the G-Function. The $G$-function is given by the Mellin-Barnes integral

$$
G_{p, q}^{m, k}\left(\lambda x \mid \begin{array}{l}
a_{p} \\
b_{q}
\end{array}\right)=\frac{1}{2 \pi i} \int_{L} \frac{\prod_{j=1}^{m} \Gamma\left(b_{j}-s\right) \prod_{j=1}^{k} \Gamma\left(1-a_{j}+s\right)}{\prod_{j=m+1}^{q} \Gamma\left(1-b_{j}+s\right) \prod_{j=k+1}^{p} \Gamma\left(a_{j}-s\right)}(\lambda x)^{s} d s
$$

where an empty product is interpreted as $1,0 \leqq m \leqq q, 0 \leqq n \leqq p$ and the parameters are such that no pole of $\Gamma\left(b_{j}-s\right), j=1,2, \cdots, m$ coincides with any pole of $\Gamma\left(1-a_{h}+s\right), h=1,2, \cdots, k$. We assume $x$ is real and the path $L$ runs parallel to the imaginary axis and is indented so that the poles of $\Gamma\left(b_{j}-s\right)$, $j=1,2, \cdots, m$, are to the right, and all the poles of $\Gamma\left(1-a_{h}+s\right), h=1$, $2, \cdots, k$, to the left of $L$. The integral converges if $p+q<2(m+k)$ and $|\arg \lambda|<(m+k-p / 2-q / 2) \pi$. For a treatment of the $G$-function, see [1, Ch. 5].

Now from $[1,10.20(3)]$ we have the expansion

$$
\begin{aligned}
x^{s} & =\Gamma(\beta-s+1) \Gamma(1-s) \\
& \times \sum_{n=0}^{\infty} \frac{(2 n+\alpha+\beta+1)(n+\beta+1)_{\alpha}}{\Gamma(n+\alpha+\beta+2-s) \Gamma(1-s-n)} R_{n}^{(\alpha, \beta)}(1 / x), \quad 1<x<\infty,
\end{aligned}
$$

uniformly for $\operatorname{Re}(s) \leqq \mu-\delta, \delta>0, \mu=\min \left(\beta+1, \beta / 2+\frac{3}{4}\right), \alpha>-1, \beta>-1$. Put (2.2) in (2.1) and integrate along the path from $\mu-\delta-i \infty$ to $\mu-\delta+i \infty$. We then get

Theorem I. Let

1. $\alpha, \beta$ and $x$ be real, $\alpha>-1, \beta>-1,1<x<\infty$.

Let a real positive $\delta$ exist such that

2. (a) $\operatorname{Re}\left(a_{j}-1\right)<\mu-\delta, j=1,2, \cdots k$; (b) $\operatorname{Re}\left(b_{j}\right)>\mu-\delta, j=1,2$, $\cdots m, \mu-\delta<1, \mu=\min \left(\beta+1, \beta / 2+\frac{3}{4}\right)$.

3. $p+q<2(m+k),|\arg \lambda|<(m+k-p / 2-q / 2) \pi, \lambda \neq 0,0 \leqq m \leqq q$, $0 \leqq k \leqq p$.

Then

$$
\begin{aligned}
G_{p, q}^{m, k}\left(\left.\lambda x_{b}^{a}\right|_{q} ^{p}\right)=\sum_{n=0}^{\infty}(2 n+\alpha+\beta+ & 1)(n+\beta+1)_{\alpha} \\
& \times G_{p+2, q+2}^{m+2, k}\left(\left.\lambda\right|_{1, \beta+1, b_{q}} ^{a_{p}, 1-n, n+\alpha+\beta+2}\right) R_{n}{ }^{(\alpha, \beta)}(1 / x) .
\end{aligned}
$$

Remark. Assumptions 2 above insure the separation of poles and specify the regions in which they lie according to the remarks surrounding (2.1). Notice, however, that poles of $\Gamma\left(b_{j}-s\right)$ may lie to the left of the contour. They may be excluded 
by indentations since they lie in a region where the series for $x^{s}$ converges uniformly, provided they do not coincide with any of the poles of $\Gamma\left(1-a_{h}+s\right)$. Hence, we may replace 2 (b) by the weaker but more complicated condition

$$
\begin{array}{rl}
2(\mathrm{~b})^{*} & 1+\delta_{j-2}-a_{h} \neq 0,-1,-2, \cdots, \\
& j=1,2, \cdots m+2, h=1,2, \cdots k, \delta_{-2}=1, \delta_{-1}=\beta+1, \delta_{j-2}=b_{j}, j>1 .
\end{array}
$$

Notice from the definition of the $G$-function that

$$
G_{p+2, q+2}^{m+2, k}\left(\left.\lambda\right|_{1, \beta+1, b_{q}} ^{a_{p}, 1-n, n+\beta+2}\right)=(-)^{n} G_{p+2, q+2}^{m+1, k+1}\left(\left.\lambda\right|_{\beta+1, b_{q}, 1} ^{1-n, a_{p}, n+\alpha+\beta+2}\right) .
$$

If $|\arg \lambda|<\frac{1}{2}(p-q+1) \pi$, an asymptotic representation for the coefficients of $R_{n}{ }^{(\alpha, \beta)}(1 / x)$ in (2.3) follows by application of a result in [1, 5.3(6)]. An ascending series representation follows when $[1,5.3(5)]$ is applied to the right-hand side of (2.4).

3. Expansion of a Confluent Hypergeometric Function. We consider the function [1, Ch. 6],

$$
(\lambda x)^{a} \psi(a, c \mid \lambda x)=\{\Gamma(a) \Gamma(\sigma)\}^{-1} G_{1,2}^{2,1}\left(\left.\lambda x\right|_{a, \sigma} ^{1}\right), \quad \sigma=a+1-c .
$$

Also, denote by $T_{n}{ }^{*}(x)$ the shifted Chebyshev polynomial

$$
T_{n}^{*}(x)=T_{n}(2 x-1)=\frac{n !}{\left(\frac{1}{2}\right)_{n}} R_{n}^{(-1 / 2,-1 / 2)}(x) .
$$

From Theorem I, we get

TheOREM II. Let

1. $1 \leqq x \leqq \infty$;

2. $\sigma \neq 0,-1,-2, \cdots ; a \neq 0,-1,-2, \cdots$;

3. $|\arg \lambda|<3 \pi / 2, \lambda \neq 0$.

Then

$$
(\lambda x)^{a} \psi(a, c \mid \lambda x)=\sum_{n=0}^{\infty} C_{n}(\lambda) T_{n}^{*}(1 / x)
$$

where

$$
C_{n}(\lambda)=\frac{\epsilon_{n}}{\pi^{1 / 2} \Gamma(a) \Gamma(\sigma)} G_{3,4}^{4,1}\left(\left.\lambda\right|_{1,1 / 2, a, \sigma} ^{1,1-n, n+1}\right), \quad \epsilon_{0}=1, \epsilon_{n}=2, n>0
$$

or

$$
C_{n}(\lambda)=\frac{\epsilon_{n}(-)^{n}}{\pi^{1 / 2} \Gamma(a) \Gamma(\sigma)} G_{2,3}^{3,1}\left(\left.\lambda\right|_{1 / 2, a, \sigma} ^{1-n, n+1}\right) .
$$

Also, if none of the quantities $\frac{1}{2}, a$ and $\sigma$ differ by an integer

$$
\begin{aligned}
& C_{n}(\lambda)=\frac{\epsilon_{n}(-)^{n}}{\pi^{1 / 2}}\left\{(a)_{-1 / 2}(\sigma)_{-1 / 2} \lambda_{2}^{1 / 2} F_{2}\left(\begin{array}{c}
n+1 / 2,-n+2,-n+1 / 2 \\
3 / 2-a, 2-\sigma
\end{array} \mid \lambda\right)\right. \\
& +\frac{\Gamma\left(\frac{1}{2}-a\right)(a)_{n}(\sigma)_{-a}}{\Gamma(n+1-a)} \lambda_{2}^{a} F_{2}\left(\begin{array}{c}
n+a,-n+a \\
a+1 / 2, a-\sigma+1
\end{array} \mid \lambda\right) \\
& \left.+\frac{\Gamma\left(\frac{1}{2}-\sigma\right)(\sigma)_{n}(a)_{-\sigma}}{\Gamma(n-\sigma+1)} \lambda_{2}^{\sigma} F_{2}\left(\begin{array}{c}
n+\sigma,-n+\sigma \\
\sigma+1 / 2, \sigma-a+1
\end{array} \mid \lambda\right)\right\},
\end{aligned}
$$


and

$$
C_{n}(\lambda) \sim \frac{\epsilon_{n}(-)^{n}(a)_{n}(\sigma)_{n}}{n !(4 \lambda)^{n}} F_{1} F_{1}\left(\begin{array}{l}
n+1 / 2, n+a, n+\sigma \\
2 n+1
\end{array} \mid-\frac{1}{\lambda}\right), \quad|\lambda| \rightarrow \infty, \quad|\arg \lambda|<\pi .
$$

Remark. Condition 1 of Theorem I is conservative. By an appeal to the convergence properties of expansions in Chebyshev polynomials [7], the range of $x$ may be extended to give condition 1 above.

Since (3.3) converges,

$$
\lim _{n \rightarrow \infty} C_{n}(\lambda)=0 .
$$

For later use, we record the fact that

$$
\lim _{x \rightarrow \infty}(\lambda x)^{a} \psi(a, c \mid \lambda x)=1, \quad|\arg \lambda|<3 \pi / 2 .
$$

4. Calculation of the Coefficients $C_{n}(\lambda)$. Let

$$
\varphi_{1, n}(\lambda)=\frac{(-)^{n}}{\epsilon_{n}} C_{n}(\lambda) .
$$

Following the method developed in [8], we can show from the representation (3.7) that $\varphi_{1, n}(\lambda)$ satisfies the recursion relation

$$
\varphi_{n}(\lambda)+\left(A_{n}+B_{n} \lambda\right) \varphi_{n+1}(\lambda)+\left(C_{n}+D_{n} \lambda\right) \varphi_{n+2}(\lambda)+E_{n} \varphi_{n+3}(\lambda)=0
$$

where

$$
\begin{aligned}
& A_{n}=(2 n+2)\left[1-\frac{\left(n+\frac{3}{2}\right)(n+a+1)(n+\sigma+1)}{(n+2)(n+a)(n+\sigma)}\right], \\
& B_{n}=D_{n}=-4(n+1) /(n+a)(n+\sigma), \\
& C_{n}=-1+[2(n+1)(2 n+3) /(n+a)(n+\sigma)], \\
& E_{n}=-(n+1)(n-a+3)(n-\sigma+3) /(n+2)(n+a)(n+\sigma) .
\end{aligned}
$$

We prove that the coefficients may be readily evaluated using (4.2) in the backward direction. This backward recursion technique has been treated by many authors [9], [10], [11], [12], [13]. The idea is as follows.

For fixed $\lambda$, arbitrary $\eta$ and $\nu$ sufficiently large set

$$
\begin{aligned}
& \varphi_{\nu}^{(\nu)}(\lambda)=\varphi_{\nu-1}^{(\nu)}(\lambda)=0, \\
& \varphi_{\nu-2}^{(\nu)}(\lambda)=\eta .
\end{aligned}
$$

The sequence $\varphi_{\nu-3}^{(\nu)}(\lambda), \cdots, \varphi_{n}{ }^{(\nu)}(\lambda), \cdots \varphi_{1}{ }^{(\nu)}(\lambda), \varphi_{0}{ }^{(\nu)}(\lambda)$ is generated from (4.2). Using (3.9) and

$$
T_{n}^{*}(0)=(-)^{n}
$$

in (3.3) we would expect that if

$$
\omega_{\nu}=\sum_{n=0}^{\nu-2} \epsilon_{n} \varphi_{n}^{(\nu)}(\lambda)
$$


then

$$
C_{n}(\lambda) \sim(-)^{n} \epsilon_{n} \varphi_{n}{ }^{(\nu)}(\lambda) / \omega_{\nu},
$$

with increasing accuracy as $\nu \rightarrow \infty$. In fact if we define

$$
\varphi_{1, n}^{(\nu)}(\lambda)=\varphi_{1,0}(\lambda) \varphi_{n}{ }^{(\nu)}(\lambda) / \varphi_{0}{ }^{(\nu)}(\lambda)
$$

we have:

Theorem III. Let $|\arg \lambda|<\pi, \lambda \neq 0$, and neither a nor $\sigma$ be a negative integer or zero. Then

$$
\lim _{\nu \rightarrow \infty} \varphi_{1, n}^{(\nu)}(\lambda)=\varphi_{1, n}(\lambda) .
$$

Proof. Denote by $\varphi_{1, n}(\lambda), \varphi_{2, n}(\lambda)$ and $\varphi_{3, n}(\lambda)$ the three linearly independent solutions of $(4.2) ; \varphi_{1, n}(\lambda)$ is the solution we wish to calculate. We may write*

$$
\varphi_{n}{ }^{(\nu)}=\xi_{1}{ }^{(\nu)} \varphi_{1, n}+\xi_{2}{ }^{(\nu)} \varphi_{2, n}+\xi_{3}{ }^{(\nu)} \varphi_{3, n}, \quad n<\nu-2,
$$

and the conditions (4.4) and (4.5) give

$$
\begin{aligned}
& 0=\xi_{1}{ }^{(\nu)} \varphi_{1, \nu}+\xi_{2}{ }^{(\nu)} \varphi_{2, \nu}+\xi_{3}{ }^{(\nu)} \varphi_{3, \nu}, \\
& 0=\xi_{1}{ }^{(\nu)} \varphi_{1, \nu-1}+\xi_{2}{ }^{(\nu)} \varphi_{2, \nu-1}+\xi_{3}{ }^{(\nu)} \varphi_{3, \nu-1}, \\
& \eta=\xi_{1}{ }^{(\nu)} \varphi_{1, \nu-2}+\xi_{2}{ }^{(\nu)} \varphi_{2, \nu-2}+\xi_{3}{ }^{(\nu)} \varphi_{3, \nu-2},
\end{aligned}
$$

where $\xi_{1}{ }^{(\nu)}, \xi_{2}{ }^{(\nu)}$ and $\xi_{3}{ }^{(\nu)}$ are independent of $n$.

$$
\begin{aligned}
& \xi_{2}{ }^{(\nu)} / \xi_{1}{ }^{(\nu)}=\gamma_{\nu}, \quad \xi_{3}{ }^{(\nu)} / \xi_{1}{ }^{(\nu)}=\delta_{\nu}, \\
& \gamma_{\nu}=\left[-\varphi_{1, \nu} \varphi_{3, \nu-1}+\varphi_{1, \nu-1} \varphi_{3, \nu}\right] / \tau_{\nu}, \\
& \delta_{\nu}=\left[-\varphi_{2, \nu} \varphi_{1, \nu-1}+\varphi_{1, \nu} \varphi_{2, \nu-1}\right] / \tau_{\nu}, \\
& \tau_{\nu}=\left[\varphi_{2, \nu} \varphi_{3, \nu-1}-\varphi_{3, \nu} \varphi_{2, \nu-1}\right] \text {. }
\end{aligned}
$$

Thus

$$
\varphi_{1, n}^{(\nu)}=\frac{\varphi_{1, n}\left\{1+\left(\gamma_{\nu} \varphi_{2, n} / \varphi_{1, n}\right)+\left(\delta_{\nu} \varphi_{3, n} / \varphi_{1, n}\right)\right\}}{\left\{1+\left(\gamma_{\nu} \varphi_{2, n} / \varphi_{1,0}\right)+\left(\delta_{\nu} \varphi_{3,0} / \varphi_{1,0}\right)\right\}} .
$$

We will show that

$$
\lim _{\nu \rightarrow \infty} \gamma_{\nu}=\lim _{\nu \rightarrow \infty} \delta_{\nu}=0 .
$$

Equation (3.8) gives

$$
\lim _{\nu \rightarrow \infty} \varphi_{1, \nu}=0 .
$$

It may be directly verified that

$$
\varphi_{2, n}={ }_{2} F_{2}\left(\begin{array}{c}
n+1 / 2,-n+1 / 2,-n+2,- \\
3 / 2-a
\end{array} \mid \lambda\right),
$$

is also a solution of (4.2). From [14] we have

$$
\varphi_{2, n}=C_{1} n^{2 / 3[a+\sigma-2]} \exp \left[\frac{3}{2} n^{2 / 3} \lambda^{1 / 3}\right]\left[1+O\left(\frac{1}{n}\right)\right], \quad|\arg \lambda|<\pi,
$$

\footnotetext{
* Henceforth we write, $\xi_{1}{ }^{(\nu)}(\lambda)=\xi_{1}^{(\nu)}, \varphi_{1, n}(\lambda)=\varphi_{1, n}$, etc.
} 


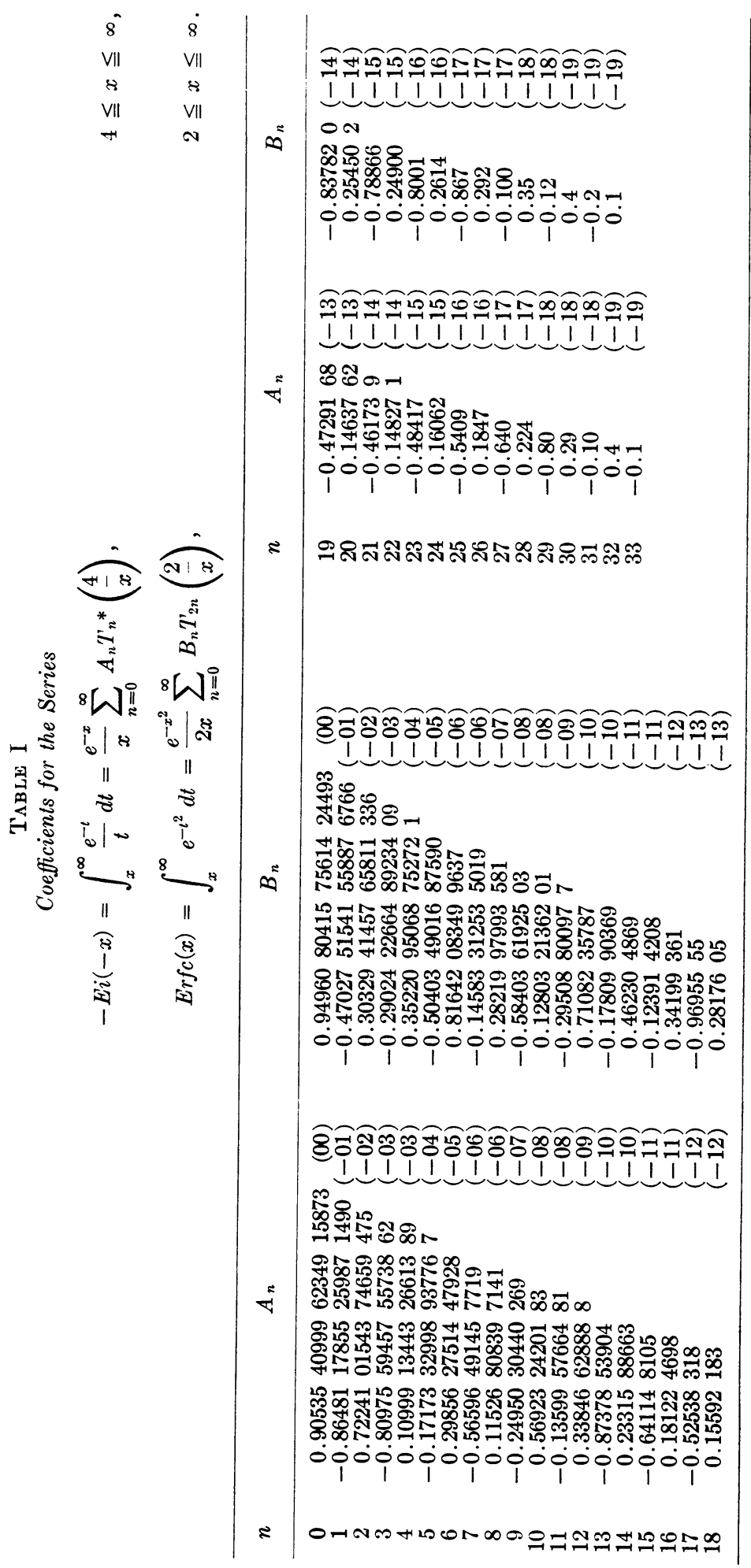




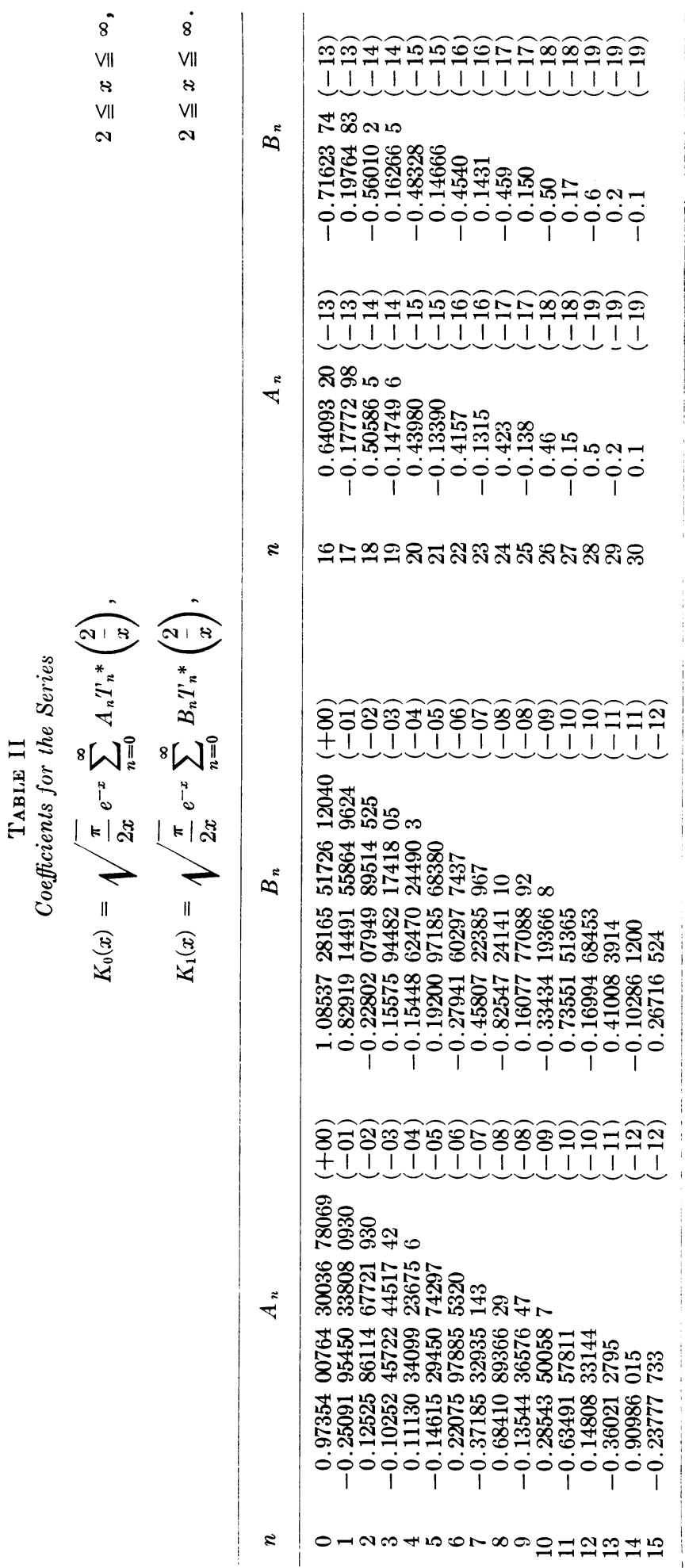




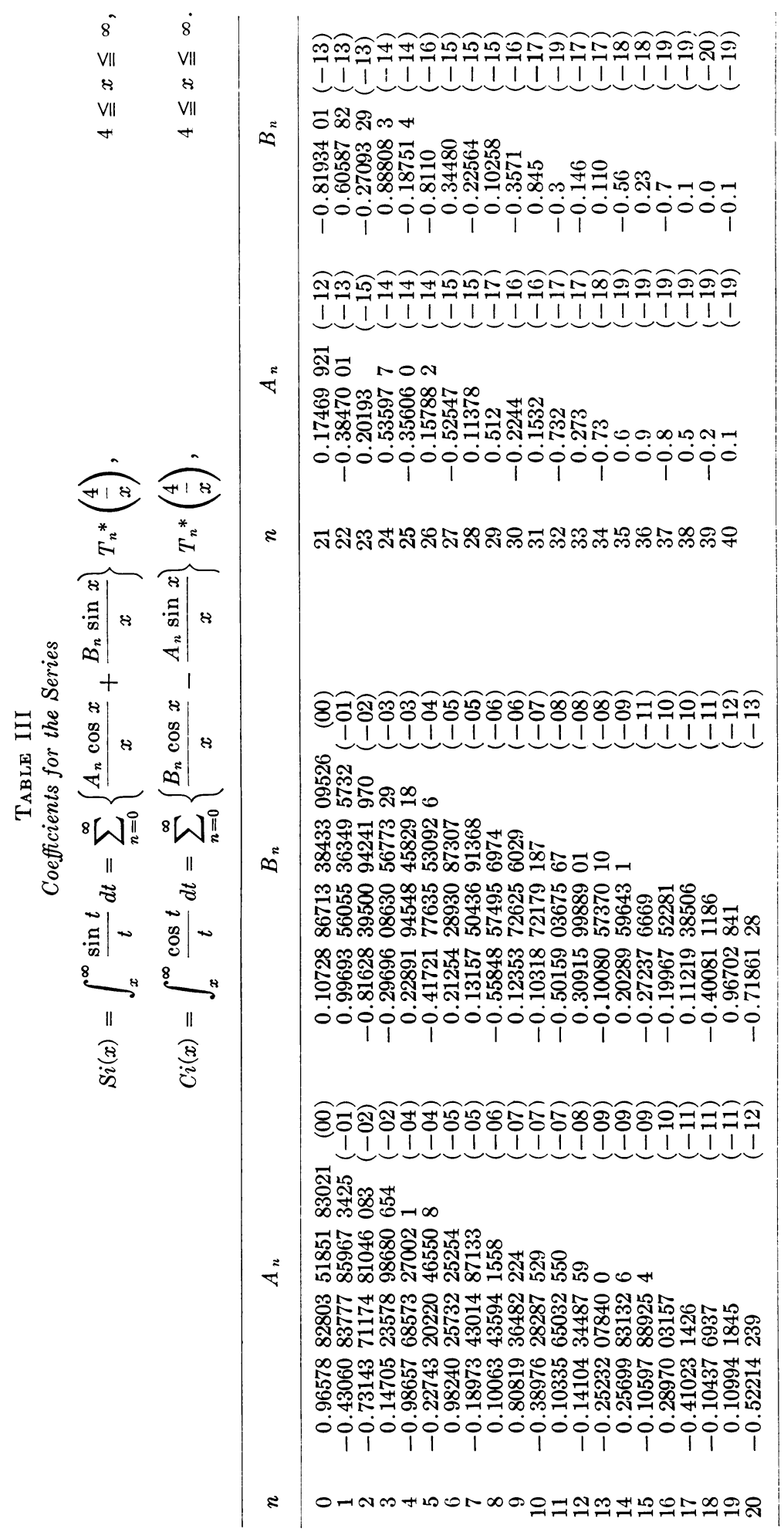


where $C_{1}$ is independent of $n$. The third linearly independent solution of (4.2) is the $L_{2,2}(-\lambda)$ term appearing in $[15,1.3 .3(15)]$ which arises in the asymptotic expansion of (4.22) for large $\lambda$. A limit process, explained in [15, 1.3.4] is used to obtain $\varphi_{3, n}$, but our discussion here is necessarily brief. We need only the estimate

$$
\varphi_{3, n}=\frac{C_{2} \Gamma(n+a-1) \Gamma(n+\sigma-1)}{(4 \lambda)^{n} n !}\left[1+O\left(\frac{1}{n^{2}}\right)\right],
$$

where $C_{2}$ is independent of $n$. Thus

$$
\lim _{\nu \rightarrow \infty}\left|\varphi_{2, \nu}\right|=\lim _{\nu \rightarrow \infty}\left|\varphi_{3, \nu}\right|=\infty .
$$

Also, from (4.23) and (4.24), we have

$$
\tau_{\nu}=-\varphi_{2, \nu} \varphi_{3, \nu}\left[1+O\left(\frac{1}{\nu}\right)\right] .
$$

Hence (4.20) is easily shown and the statement (4.10) follows from (4.19).

5. Tables. Tables I-III contain coefficients to $20 \mathrm{D}$ for the expansions of several important cases of the confluent hypergeometric function [1, 6.9]. Coefficients corresponding to different ranges of the independent variable as well as those for other functions, e.g., $J_{\nu}(x)$ and $Y_{\nu}(x)$, are under construction and the present tables are selected examples only. The expansions are readily evaluated using a nesting procedure described in [4], [7]. For similar expansions, see [7], and for many Chebyshev expansions of functions over a finite interval, see [2]-[6] and the references given there. The number in parenthesis after each entry in the tables is the power of 10 by which the entry is to be multiplied.

6. Acknowledgment. This paper covers research initiated by the Applied Mathematics Laboratory, David W. Taylor Model Basin, Washington, D. C., under Contract No. Nonr-2638 (00)(X).

The authors acknowledge with thanks the valuable assistance of our colleagues, particularly the suggestions of Jerry Fields. The coefficients tabulated in Section V were computed on the IBM 1620, and the authors are indebted to the experience and skill of Dean Lawrence, who supervised the calculations.

Midwest Research Institute

Kansas City, Missouri

1. A. Erdélyi, W. Magnus, F. Oberhettinger, \& F. G. Tricomi, Higher Transcendental Functions, v. 1 and 2, McGraw-Hill, New York, 1953.

2. Jet Wimp, "Polynomial approximations to integral transforms," Math. Comp., v. 15, p. $174-178$.

3. JeRRY L. Fields, \& Jet Wimp, "Expansions of hypergeometric functions in hypergeometric functions," Math. Comp., v. 15, p. 390-395.

4. Jeт Wimp, "Polynomial expansions of Bessel functions and some associated functions," Math. Comp., v. 16, p. 446-458.

5. Jet Wimp, \& Y. L. Luke, "Expansion formulas for generalized hypergeometric functions," to appear.

6. Jerry L. Fields, \& Jet Wimp, "Basic series corresponding to a class of hypergeometric polynomials," Proc. Cambridge Philos. Soc., forthcoming.

7. C. W. Clenshaw, Chebyshev Series for Mathematical Functions, Math. Tables, v. 5, National Physical Laboratory, London, 1962.

8. Earl Rainville, Special Functions, Macmillan, New York, 1960. 
9. M. Goldstein, \& R. M. Thaler, "Recurrence techniques for the calculation of Bessel functions," $M T A C$, v. 13, p. 102-108.

10. F. J. Corbató, \& J. L. URETsky, "Generation of spherical Bessel functions in digital computers," J. Assoc. Comput. Mach., v. 6, p. 366-375.

11. I. A. StEgUn, \& M. ABramowitz, "Generation of Bessel functions on high speed computers," MTAC, v. 11, p. 255-257.

12. W. GAUTSCHI, "Recursive computation of the repeated integrals of the error function," Math. Comp., v. 15, p. 227-232.

13. W. GAUTSCHI, "Recursive computation of certain integrals," J. Assoc. Comput. Mach. v. 8 , p. $21-40$.

14. JerRY L. Fields, \& Y. L. Luke, "Asymptotic expansions of a class of hypergeometric polynomials with respect to the order, II,"J.Math. Anal. Appl., forthcoming.

15. Y. L. LUKE, Integrals of Bessel Functions, McGraw-Hill, New York, 1962. 